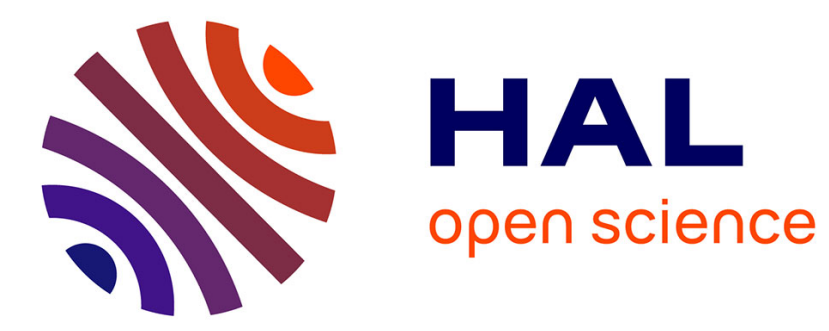

\title{
First-principles calculations of momentum distributions of annihilating electron-positron pairs in defects in UO_2
}

\author{
Julia Wiktor, Gérald Jomard, Marc Torrent, M. Bertolus
}

\section{To cite this version:}

Julia Wiktor, Gérald Jomard, Marc Torrent, M. Bertolus. First-principles calculations of momentum distributions of annihilating electron-positron pairs in defects in UO_2. Journal of Physics: Condensed Matter, 2017, 29 (3), pp.035503. 10.1088/1361-648X/29/3/035503 . cea-02389430

\section{HAL Id: cea-02389430 https://hal-cea.archives-ouvertes.fr/cea-02389430}

Submitted on 2 Dec 2019

HAL is a multi-disciplinary open access archive for the deposit and dissemination of scientific research documents, whether they are published or not. The documents may come from teaching and research institutions in France or abroad, or from public or private research centers.
L'archive ouverte pluridisciplinaire HAL, est destinée au dépôt et à la diffusion de documents scientifiques de niveau recherche, publiés ou non, émanant des établissements d'enseignement et de recherche français ou étrangers, des laboratoires publics ou privés. 


\title{
First-principles calculations of momentum distributions of annihilating electron-positron pairs in defects in $\mathrm{UO}_{2}$
}

\author{
Julia Wiktor, ${ }^{1}$ Gérald Jomard, ${ }^{1}$ Marc Torrent, ${ }^{2}$ and Marjorie Bertolus ${ }^{1}$ \\ ${ }^{1}$ CEA, DEN, DEC, Centre de Cadarache, 13108, Saint-Paul-lez-Durance, France \\ ${ }^{2}$ CEA, DAM, DIF, F-91297 Arpajon, France
}

\begin{abstract}
We perform first-principles calculations of momentum distributions of annihilating electronpositron pairs in vacancies in uranium dioxide. We take into account the full atomic relaxation effect (due to both electronic and positronic forces) and use self-consistent two-component density functional theory schemes. We present one-dimensional momentum distributions (Doppler-broadened annihilation radiation line shapes) along with line-shape parameters $S$ and $W$. The effect of krypton incorporation in the vacancy is also considered and it is shown that it should be possible to observe the fission gas incorporation in defects in $\mathrm{UO}_{2}$ using positron annihilation spectroscopy.
\end{abstract}

\section{INTRODUCTION}

Uranium dioxide $\left(\mathrm{UO}_{2}\right)$ is currently the most widely used fuel material in pressurized water reactors (PWR). During the reactor operation the fission of actinide nuclei causes the creation of large amounts of defects, what leads to the evolution of the physical and chemical properties of $\mathrm{UO}_{2}$. To understand the behavior of the fuel material during reactor operation, it is essential to study the effects of the irradiation on the thermomechanical and thermochemical properties of $\mathrm{UO}_{2}$ caused by defects creation and their interaction with fission products.

Positron annihilation spectroscopy (PAS) $)^{1,2}$ is one of the non-destructive methods that can be used to study vacancies. There are two positron annihilation features that allow one to characterize defects in solids: the positron lifetime, mostly sensitive to the open volume of defects, and the Doppler broadening of the annihilation radiation, carrying information on the chemical environment in which positrons annihilate. Since PAS results do not provide a direct link between the signal and the type of the defect, comparisons with other experimental techniques or with electronic structure calculations are often required in the interpretation of experimental data.

In a recent study we performed fully self-consistent calculations of positron lifetimes for defects in uranium dioxide $^{3}$ and used the computational results to identify defects in $\alpha$-irradiated $\mathrm{UO}_{2}$ samples. We observed that in some cases different vacancies exhibit similar positron lifetimes and that it can be difficult to distinguish them based on this characteristic itself. In the present work we perform calculations of the second positron annihilation characteristic, the Doppler broadening of the annihilation radiation, which is often measured in experimental positron studies on $\mathrm{UO}_{2}{ }^{4-9}$ and can bring complementary information on defects. This paper is organized as follows: In Sec. II we briefly present the computational methods. In Sec. III we present the calculated Doppler spectra and $S$ and $W$ parameters and discuss the results. Finally, we conclude the paper in Sec. IV.

\section{METHODS}

We use the implementation of the two-component density functional theory (TCDFT) $)^{1,10,11}$ within the projector augmented-wave (PAW) ${ }^{12-15}$ framework, available in the ABINIT code ${ }^{16-18}$. The methods are described in Ref. 19 and we only recall them briefly here.

In this work we use the so-called 'state-dependent' 20 scheme to calculate the momentum distributions of the annihilating electron-positron pairs. In this method the momentum distribution $\rho(\mathbf{p})$ is expressed as:

$$
\rho(\mathbf{p})=\pi r_{e}^{2} c \sum_{i j} \gamma_{j}\left|\int \mathrm{d} \mathbf{r} e^{-i \mathbf{p} \cdot \mathbf{r}} \Psi^{+}(\mathbf{r}) \Psi_{j}^{-}(\mathbf{r})\right|^{2},
$$

where $\Psi^{+}(\mathbf{r})$ is the positron wavefunction, $\Psi_{j}^{-}(\mathbf{r})$ is the wavefunction of the electron state $j, \mathbf{p}$ is a given momentum, $r_{\mathrm{e}}$ is the classical electron radius and $c$ is the speed of light. In the above equation $\gamma_{j}$ is equal to $\lambda_{j} / \lambda_{j}^{\mathrm{IPM}}$, where $\lambda_{j}$ is the total annihilation rate calculated for the electronic state $j$,

$$
\lambda_{j}=\pi r_{\mathrm{e}}^{2} c \int \mathrm{d} \mathbf{r} n_{j}^{-}(\mathbf{r}) n^{+}(\mathbf{r}) \gamma\left(n^{-}\right),
$$

and $\lambda_{j}^{\mathrm{IPM}}$ is the annihilation rate calculated for the same state within the IPM, using $\gamma=1 . n^{+}(\mathbf{r})$ is the positron density and $n_{j}^{-}(\mathbf{r})$ is the density corresponding to the electron state $j$.

To calculate the Doppler spectra of the perfect lattice we use the conventional (CONV) scheme, in which the LDA zero-positron density limit of the electron-positron correlation functional, parametrized by Boroński and Nieminen ${ }^{11}$ using the data provided by Arponen and Pajanne $^{21}$, is taken. In momentum distribution calculations for vacancies, we perform fully self-consistent calculations, using a full LDA electron-positron correlation functional provided by Puska, Seitsonen, and Nieminen ${ }^{22}$ and an enhancement factor depending on both the electron and the positron densities, $g\left(n^{-}, n^{+}\right)$. This method corresponds to the so-called PSN scheme, in which case Eq. (2) is replaced by:

$$
\lambda_{j}=\pi r_{\mathrm{e}}^{2} c \int \mathrm{d} \mathbf{r} n_{j}^{-}(\mathbf{r}) n^{+}(\mathbf{r}) g\left(n^{-}, n^{+}\right),
$$


The calculations are performed as self-consistent loops on the electronic and positronic densities: during each subloop, one of the two densities is kept constant while the other is being converged. In the calculations we allow the atomic positions to relax, according to the electronic and positronic forces, since it has been shown that the relaxation effect can affect strongly the calculated positron annihilation characteristics ${ }^{23-25}$.

Both electronic and positronic wavefunctions are described using the same mixed basis (planewaves and atomic orbitals) in the framework of the PAW method. The PAW data sets are generated using a modified version of the ATOMPAW code ${ }^{14}$. For oxygen we included 8 electrons $(1 s, 2 s$ and $2 p)$ and for uranium 14 electrons $(5 f, 6 s, 6 p, 6 d$ and $7 s)$ in the valence state. Additionally, to achieve a correct description of the positron wavefunction, we add $5 s$ and $5 p$ states in the U basis set (see Ref. ${ }^{19}$ for the explanation of the dataset generation and discussion on the PAW data set completeness for the positron description).

We use the generalized gradient approximation (GGA) as parametrized by Perdew, Burke, Ernzerhof $(\mathrm{PBE})^{26}$ to describe the exchange-correlation interactions. Moreover, a Hubbard-like term $(U)$ is added in order to take into account the strong correlations between the $5 f$ electrons of the uranium atoms. The Liechtenstein scheme ${ }^{27}$ of the DFT $+U$ method is used. The values of the $U$ and $J$ parameters are set to $4.5 \mathrm{eV}$ and $0.51 \mathrm{eV}$ respectively, in agreement with earlier DFT $+U$ calculations ${ }^{28-32}$ and the values extracted from experiments ${ }^{33}$. In order to avoid the convergence to one of the numerous metastable states yielded by the DFT $+U$ method and ensure that the ground state is reached, we used the occupation matrix control scheme $\mathrm{s}^{31,34,35}$. We consider a $1 \mathbf{k}$ antiferromagnetic ordering, which is an approximation of the noncollinear $3 \mathbf{k}$ order, since the two exhibit only small differences in energy ${ }^{36}$. We did not take into account the spin-orbit coupling (SOC) due to the too high computational cost. Extensive investigations of the SOC influence on the properties of actinide compounds ${ }^{37,38}$ suggest that it does not affect the properties of defects ${ }^{39}$.

Calculations for defects are performed using 96 atom supercells $(2 \times 2 \times 2$ repetitions of the fluorite unit cell). We use the equilibrium lattice parameters of $\mathrm{UO}_{2}$ yielded by the set of parameters described above, $a=b=5.57 \AA$ and $c=5.49 \AA$. The positron wavefuntion and density are calculated at two k-points, the $\Gamma$ point and another one chosen to lie on the edge of the Brilloun zone, to avoid the delocalization of the positron due to the small supercell, as proposed by Korhonen et al. ${ }^{40}$. The same k-points are used in the electronic calculations. The momentum distributions have been calculated using the wavefunctions corresponding to the $\Gamma$ point only. We used the cut-off energy of $680 \mathrm{eV}$, since we found it was enough to obtain Doppler spectra converged up to $40 \mathrm{mrad}$. The atomic relaxation is performed until the forces acting on atoms become smaller than $0.03 \mathrm{eV} / \AA$.

For comparison with experiments we calculate one- dimensional projections (Doppler spectra) of the threedimensional momentum densities in three different directions. This is done by integrating the momentum distribution along two remaining directions, as:

$$
\rho\left(p_{z}\right)=\iint \mathrm{d} p_{x} \mathrm{~d} p_{y} \rho(\mathbf{r}) .
$$

In the present study we calculate projections in three different directions and average the results. To obtain the best quality of the Doppler spectra, we choose $p_{z}$ to be normal to the dense (001), (011) and (111) planes.

Additionally, to mimic the finite resolution of experimental measurements we convolve the theoretical results with a Gaussian function with the FWHM (full width at half maximum) equal to $4.7 \mathrm{mrad}$. We further interpolate the one-dimensional spectra on a grid with $0.1 \mathrm{mrad}$ spacing and normalize the spectra to unity.

\section{RESULTS AND DISCUSSION}

\section{A. Comparison of calculation schemes}

In this work we use the PSN scheme to calculate the momentum distributions of annihilating pairs in vacancies in $\mathrm{UO}_{2}$. We decided to study, however, the effect of the choice of the calculation method on the Doppler spectra of defects in $\mathrm{UO}_{2}$. We perform, therefore, calculations using two other schemes, CONV and GGGC (see for instance Ref. ${ }^{22}$ or $^{19}$ for the description of various schemes). In Fig. 1 we compare the ratio curves (normalized Doppler spectrum of the defect divided by the normalized Doppler spectrum of the lattice) calculated for the uranium monovacancy using the CONV, GGGC and PSN schemes. The vacancy was relaxed using the PSN and GGGC schemes. In the case of the CONV method we compare results calculated first for unrelaxed atomic positions and then taking the relaxed positions from the GGGC calculation. We can observe that the general shapes of the ratio curves calculated using different schemes are similar. For all methods a peak at $p=0$ is observed, with GGGC yielding the largest amplitude and the CONV method with unrelaxed positions the lowest. A second peak can be observed around $p=15 \mathrm{mrad}$, while around $p=27 \mathrm{mrad}$ a shoulder or a peak, depending on the method used, appears.

The effect of the atomic relaxation on the calculated ratio curve can be analyzed by comparing the results obtained using the CONV method for two different geometries (blue lines in Fig. 1). We can observe that when the uranium vacancy relaxes outwards, the low-momentum contribution increases, since the electronic density moves further from the maximum of the positron density. At the same time, the ratio curve at higher momenta decreases.

The localized shape of the ratio curve calculated using the GGGC scheme is consistent with the overestimation of the positron localization yielded by this method, 
which was already discussed in previous studies ${ }^{3,22,23,25}$. As for the PSN scheme, the ratio curve obtained using this method is the flattest. Additionally, we can see that at low momenta the results yielded by this method are in good agreement with the ones calculated using the CONV scheme in the relaxed geometry. The agreement is, however, not as good above $p=10 \mathrm{mrad}$. In the calculations presented below, we use the PSN scheme since this method yields the best description of the positron localization in the defect.

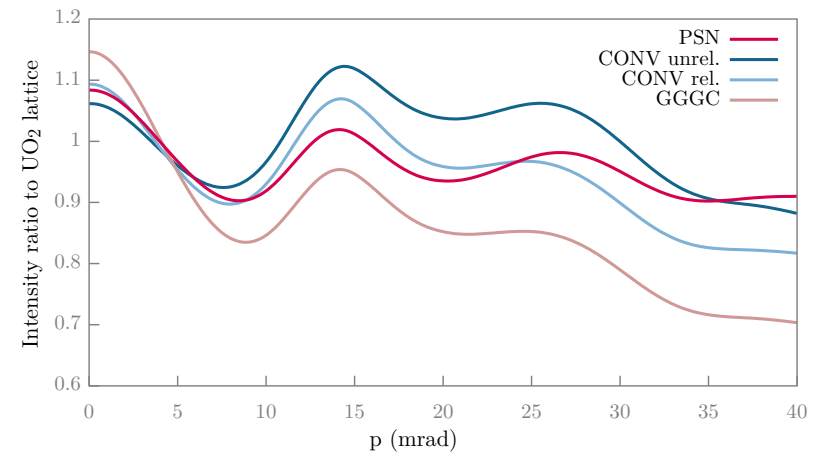

FIG. 1. (Color online) Ratio curves of the calculated momentum distributions of annihilating electron-positron pairs in $\mathrm{V}_{\mathrm{U}}$ using different schemes. All spectra are convoluted with a Gaussian function with FWHM=4.7 mrad and divided by the lattice spectrum.

\section{B. Vacancies in $\mathrm{UO}_{2}$}

We performed calculations of the momentum distributions for several vacancy defects in $\mathrm{UO}_{2}$. The ratio curves found for neutral $V_{U}, V_{U}+V_{O}$, the three configurations of $\mathrm{V}_{\mathrm{U}}+2 \mathrm{~V}_{\mathrm{O}}$ and one configuration of $2 \mathrm{~V}_{\mathrm{U}}+4 \mathrm{~V}_{\mathrm{O}}$ vacancies are presented in Fig. 2. We can see that all the calculated ratio curves are rather similar, with a maximum at $p=0$ and two peaks, one around $p=15 \mathrm{mrad}$ and one around $p=27 \mathrm{mrad}$. The curve calculated for the neutral $2 \mathrm{~V}_{\mathrm{U}}+4 \mathrm{~V}_{\mathrm{O}}$ complex is the most distinct from the others, it is more flat and has much lower values at high momenta. The similarity between for instance the monovacancy and the trivacancies can be explained by the fact, that while oxygen -2 ions are removed from the neighborhood of $\mathrm{V}_{\mathrm{U}}$, the remaining ones can attract the positron and shift its density toward them (see positron isodensities in Ref. 3). As a result, even though there are less oxygen atoms surrounding the positron, the annihilation rate with the remaining ones increases and the two effects cancel each other out, leading to rather small changes in the ratio curves. Even though it will be probably possible to observe these changes in the $S$ and $W$ parameters, it could be difficult to distinguish defects based on them.

In the study of the positron lifetimes ${ }^{3}$ we observed that the positron localization in the hexavacancy depends on

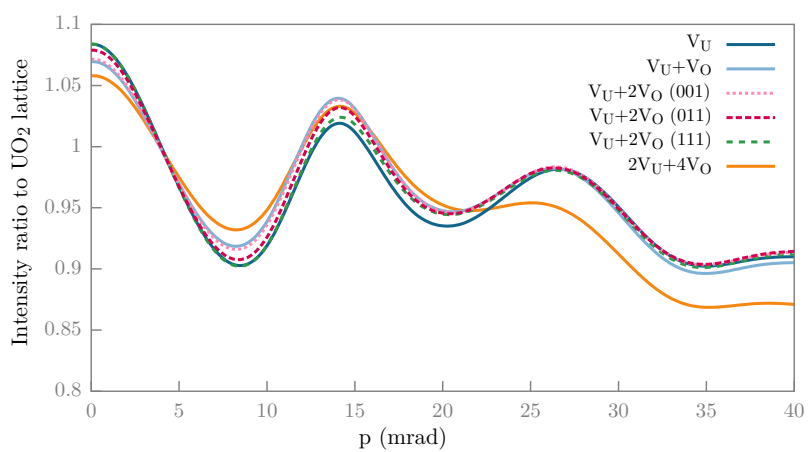

FIG. 2. (Color online) Ratio curves of the calculated momentum distributions of annihilating electron-positron pairs in various defects in $\mathrm{UO}_{2}$. All spectra are convoluted with a Gaussian function with FWHM=4.7 mrad and divided by the lattice spectrum.

the charge state of this defect. In the $\left(2 \mathrm{~V}_{\mathrm{U}}+4 \mathrm{~V}_{\mathrm{O}}\right)^{2-}$ complex the positron density was centered between the uranium sites and not inside them, as in the other defects (see Ref. 3). We performed, therefore, a momentum distribution calculation for this defect and present it in Fig. 3d, along with the ratio curves of the $V_{O}$ and $\mathrm{V}_{\mathrm{U}}$ monovacancies. In the negative hexavacancy, the positron occupies a large open volume, which is reflected in the high value of the maximum at $p=0$, around 1.2 . This is consistent with the long positron lifetime (365 ps) calculated for this defect. The curve at higher momenta has a shape similar to that of $\mathrm{V}_{\mathrm{U}}$, peaks are observed around $p=15$ and $p=27 \mathrm{mrad}$. However, the absolute values at high momenta between 10 and $30 \mathrm{mrad}$ are closer to those calculated for $\mathrm{V}_{\mathrm{O}}$.

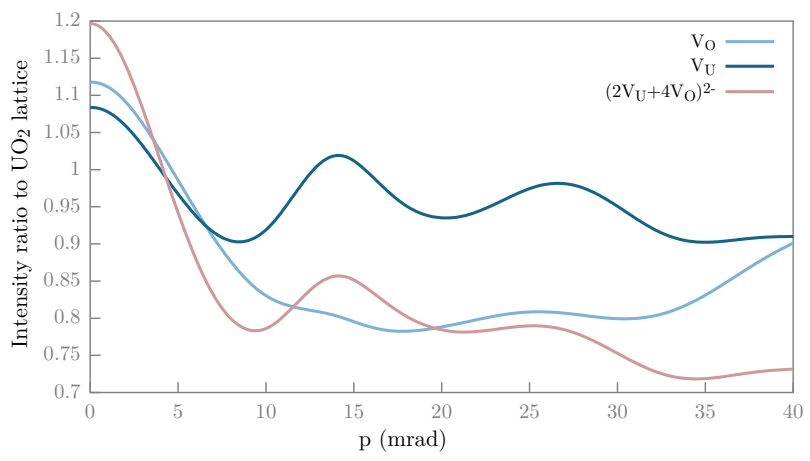

FIG. 3. (Color online) Ratio curves of the calculated momentum distributions of annihilating electron-positron pairs in various defects in $\mathrm{UO}_{2}$. All spectra are convoluted with a Gaussian function with FWHM=4.7 mrad and divided by the lattice spectrum. 


\section{Effect of the charge state}

We verified the effect of the charge state on the momentum distribution for the uranium monovacancy. In Fig. 4 we present the ratio curves calculated for $\mathrm{V}_{\mathrm{U}}$ and $\mathrm{V}_{\mathrm{U}}^{4-}$. There are only slight differences between the two charge states. For the negative monovacancy we can observe a small increase in the maximum at $p=0$ and a decrease for momenta above $p=5 \mathrm{mrad}$. These results suggest that the change of the charge state has an almost negligible effect on the ratio curves, unless it changes the site at which the positron is localized, like in the case of the hexavacancy discussed before.

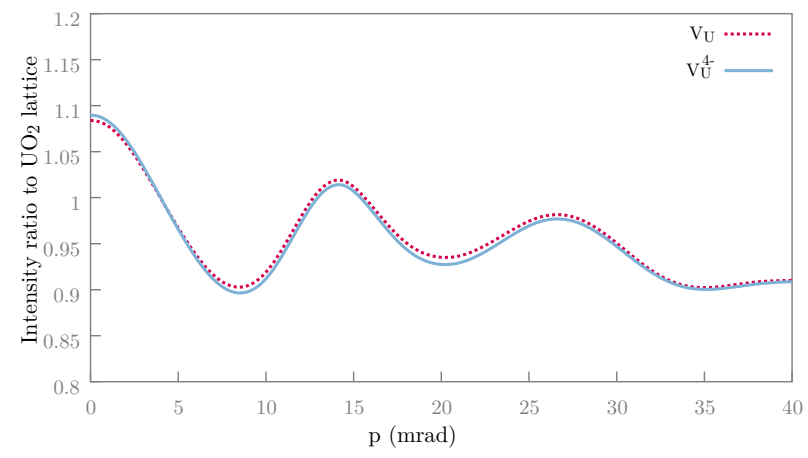

FIG. 4. (Color online) Ratio curves of the calculated momentum distributions of annihilating electron-positron pairs in $\mathrm{V}_{\mathrm{U}}$ and $\mathrm{V}_{\mathrm{U}}^{4-}$. All spectra are convoluted with a Gaussian function with FWHM=4.7 mrad and divided by the lattice spectrum.

\section{Krypton incorporation}

Incorporation of gaseous fission products in vacancies in $\mathrm{UO}_{2}$ is an important issue in the studies of fuel behavior under irradiation. The momentum distribution of the annihilation radiation is especially sensitive to the chemical environment of the defect. We have studied, therefore, the effect of a $\mathrm{Kr}$ atom on the Doppler spectrum of $\mathrm{V}_{\mathrm{U}}+2 \mathrm{~V}_{\mathrm{O}}(110)$. The calculated ratio curve is presented in Fig. 5 and the positron isodensity in this defect in Fig. 6. We can see that the Kr incorporation has a significant effect on the ratio curve. The maximum at $p=0$ is decreased, since krypton increases the electron density and decreases the free volume available to the positron, as seen in Fig. 5. Additionally, the peak around $p=15 \mathrm{mrad}$ decreases and the one around $p=27 \mathrm{mrad}$ increases. The high sensitivity of the momentum distribution of the annihilation radiation to the presence of krypton atoms suggests that such measurement can be very useful in studies of fission products incorporation in $\mathrm{UO}_{2}$.

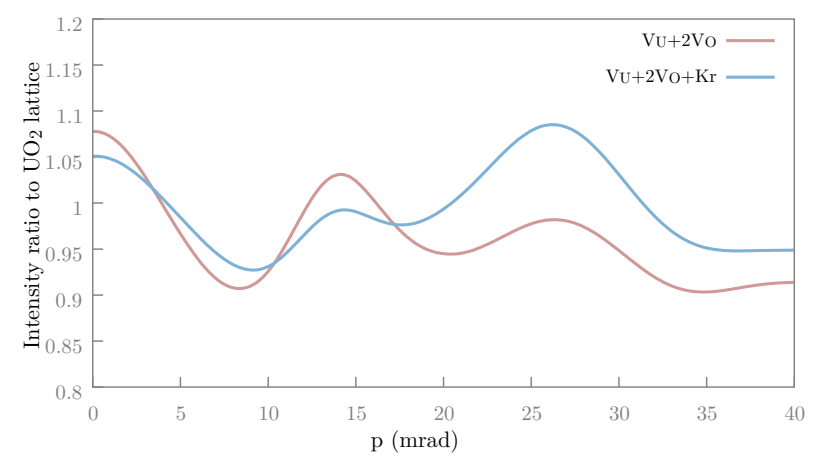

FIG. 5. Ratio curves of the calculated momentum distributions of annihilating electron-positron pairs in the $\mathrm{V}_{\mathrm{U}}+2 \mathrm{~V}_{\mathrm{O}}(110)$ trivacancy (Bound Schottky Defect), empty and containing one Kr atom. All spectra are convoluted with a Gaussian function with FWHM=4.7 mrad and divided by the lattice spectrum.

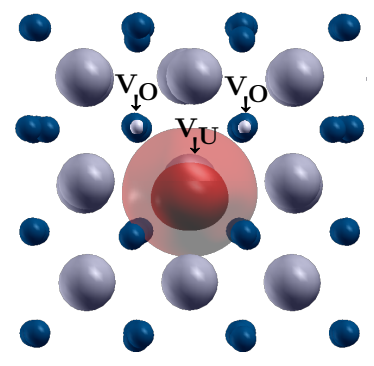

(a)

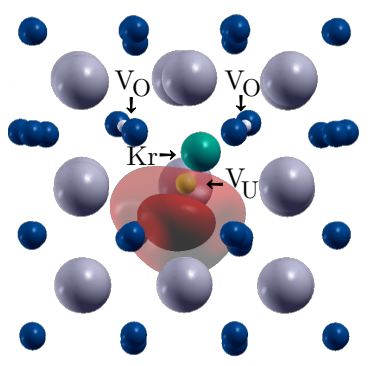

(b)
FIG. 6. (Color online) Positron isodensities (70\% of the maximum density - solid and $30 \%$ - transparent), in red, found in the $\mathrm{V}_{\mathrm{U}}+2 \mathrm{~V}_{\mathrm{O}}(110)$ defect, empty (a) and containing a $\mathrm{Kr}$ atom (b). Uranium atoms are presented in gray, oxygen atoms in blue and krypton in green. White and yellow spheres represent oxygen and uranium vacancies, respectively. Figures were generated using the XCRYSDEN ${ }^{41,42}$ program.

\section{E. $S$ and $W$ parameters}

In experimental PAS studies integrated low- and highmomentum contributions to the momentum distribution are often considered. These contributions are calculated as so-called $S$ (low-momentum) and $W$ (highmomentum) parameters, being defined, respectively, as the ratio of counts in the regions close to and far from the $511 \mathrm{keV}$ value to the total number of the counts. These parameters are usually presented as values relative to the perfect lattice and defined as:

$$
S_{\text {rel }}=\frac{S_{\text {defect }}}{S_{\text {lattice }}}
$$

and

$$
W_{\text {rel }}=\frac{W_{\text {defect }}}{W_{\text {lattice }}}
$$


We calculated the $S_{\text {rel }}$ and $W_{\text {rel }}$ parameters for the considered vacancies in $\mathrm{UO}_{2}$, using the integration windows of $S \in(0,2.8) \mathrm{mrad}$ and $W \in(10.61,26.35) \mathrm{mrad}$, as in Ref. ${ }^{6}$, and present them in Fig. 7 . We can see that the points corresponding to $\mathrm{V}_{\mathrm{U}}, \mathrm{V}_{\mathrm{U}}+\mathrm{V}_{\mathrm{O}}$, the three configurations of $\mathrm{V}_{\mathrm{U}}+2 \mathrm{~V}_{\mathrm{O}}$ and neutral $2 \mathrm{~V}_{\mathrm{U}}+4 \mathrm{~V}_{\mathrm{O}}$ are close to each other. However, they could probably be distinguished in high precision experimental measurements. The points corresponding to $\mathrm{V}_{\mathrm{O}}$ and to negative $2 \mathrm{~V}_{\mathrm{U}}+4 \mathrm{~V}_{\mathrm{O}}$ lay far from the other points, hence could be easily identified if detected in Doppler broadening measurements. By comparing the $S_{\text {rel }}$ and $W_{\text {rel }}$ parameters calculated for the empty $\mathrm{V}_{\mathrm{U}}+2 \mathrm{~V}_{\mathrm{O}}(110)$ defect and when containing a $\mathrm{Kr}$ atom, we can conclude that for the set of integration windows used, the krypton incorporation leads to a clear shift in the measured signal, which should be easily observed in experiments.

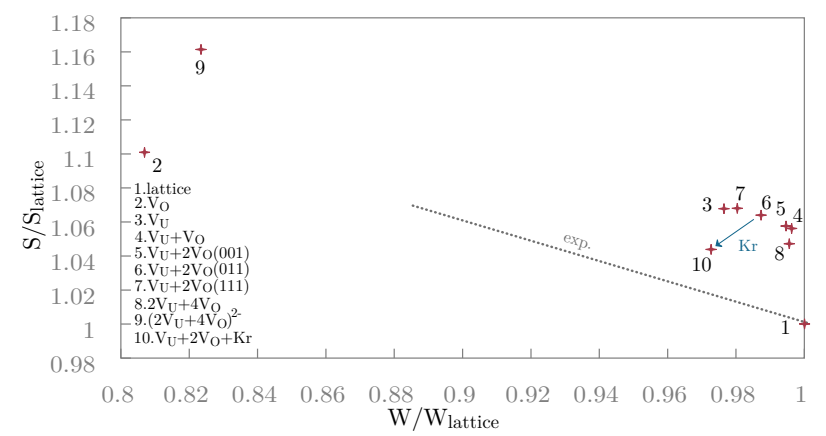

FIG. 7. (Color online) $S_{\text {rel }}$ parameter as a function of the $W_{\text {rel }}$ parameter plotted for various defects in $\mathrm{UO}_{2}$, using windows $S \in(0,2.8) \mathrm{mrad}$ and $W \in(10.61,26.35) \mathrm{mrad}$. The results were obtained using spectra convoluted with a Gaussian function with $\mathrm{FWHM}=4.7 \mathrm{mrad}$. The arrow indicates the effect of krypton incorporation. The dotted line indicates the trend in the experimental measurements presented in Ref. 6.

\section{F. Comparison with experiments}

In Fig. 7 we compare the calculated relative $S$ and $W$ parameters with the trend in measured parameters observed in Ref. 6 (dotted line). In this study $\mathrm{UO}_{2}$ samples were irradiated with electrons with various energies $(1$ and $2.5 \mathrm{MeV})$ at various fluences $\left(5 \times 10^{17} \mathrm{~cm}^{-2}\right.$ and $\left.5 \times 10^{18} \mathrm{~cm}^{-2}\right)$. The positron lifetime observed in these samples was equal to $310 \pm 5$ ps. We can clearly see that there is no agreement between the calculated and measured $S_{\text {rel. }}$ and $W_{\text {rel. parameters. We suppose that it is }}$ due to the fact that the comparison between the calculated and experimental $S_{\text {rel. }}$ and $W_{\text {rel. }}$ is only meaningful if the reference samples, considered as free of defects, exhibit positron annihilation characteristic close to those of a perfect lattice. The uranium dioxide samples taken as reference in Ref. 6 (annealed during 24 hours at $1700^{\circ} \mathrm{C}$ under $\mathrm{ArH}_{2}$ ) are hyper-stoichiometric and probably con- tain nonnegligible concentrations of defects, such as negative oxygen interstitials. These defects, even though having positron lifetimes close to that of the lattice, can give rise to significantly different momentum distributions of annihilating positron-electron pairs. This means that the denominators adopted in Eq. (5) and (6) in the present work and in the experimental study do not correspond. It is worth noting that the comparison between the absolute values of $S$ and $W$ would not solve this problem, since they depend strongly on, for instance, the detector geometry, resolution and calibration and, therefore, are meaningless ${ }^{2}$. We conclude that further experimental studies, with a careful control of the defects concentration in the reference samples and their stoichiometry, could enable an insightful comparison with calculations and a more advanced interpretation of the Doppler broadening measurements on $\mathrm{UO}_{2}$.

\section{CONCLUSIONS}

We performed first-principles calculations of momentum distribution of the annihilation radiation for various vacancy defects in uranium dioxide. Self-consistent twocomponent density functional theory schemes were used and the full atomic relaxation (due to both electronic and positronic densities) was taken into account. We studied the effect of the choice of the calculation scheme, charge state and krypton incorporation on the calculated ratio curves.

We observed that in the case of the majority of considered vacancy defects, except for $\mathrm{V}_{\mathrm{O}}$ and $\left(2 \mathrm{~V}_{\mathrm{U}}+4 \mathrm{~V}_{\mathrm{O}}\right)^{2-}$, calculations yield rather similar relative $S$ and $W$ parameters and hence they could only be distinguished in high precision experimental measurements. In the case of the uranium monovacancy we observed a negligible effect of the charge state on the calculated ratio curve. Our calculations for the bound Schottky defect containing a krypton atom indicate that the incorporation of this fission product affects strongly the Doppler spectrum of the defect and, therefore, could be successfully studied using PAS.

We did not observe a clear agreement between the calculated and measured $S_{\text {rel }}$ and $W_{\text {rel }}$ parameters. We suppose that this can be related to the fact that the reference samples may contain nonnegligible concentrations of negative oxygen interstitials. Additional experiments on samples with controlled stoichiometry could be performed to verify the influence of the reference data on the $S(W)$ plots.

\section{ACKNOWLEDGMENTS}

The authors are grateful to Marie-France Barthe and Ilja Makkonen for fruitful discussions. This research was supported by the basic research program on nuclear materials of the Nuclear Energy Division at CEA, RCOMB. 
This work was partly performed using HPC resources

from GENCI-CCRT (Grant x2015096008).

1 M. J. Puska and R. M. Nieminen, Rev. Mod. Phys. 66, 841 (1994).

${ }^{2}$ F. Tuomisto and I. Makkonen, Rev. Mod. Phys. 85, 1583 (2013), URL http://link.aps.org/doi/10.1103/ RevModPhys.85.1583.

3 J. Wiktor, M.-F. Barthe, G. Jomard, M. Torrent, M. Freyss, and M. Bertolus, Phys. Rev. B 90, 184101 (2014).

4 M.-F. Barthe, P. Desgardin, G. Blondiaux, S. Guilbert, H. Labrim, T. Sauvage, G. Carlot, P. Garcia, and J. P. Piron, in Mater. Sci. Forum (2004), vol. 445, p. 48.

${ }^{5}$ H. Labrim, M.-F. Barthe, P. Desgardin, T. Sauvage, G. Blondiaux, C. Corbel, and J. P. Piron, App. Surf. Sci. 252, 3256 (2006).

6 M.-F. Barthe, H. Labrim, A. Gentils, P. Desgardin, C. Corbel, S. Esnouf, and J. P. Piron, Phys. Status Solidi C 4, 3627 (2007).

7 H. Labrim, M.-F. Barthe, P. Desgardin, T. Sauvage, C. Corbel, G. Blondiaux, and J. P. Piron, Nucl. Instrum. Meth. B 261, 883 (2007).

8 N. Djourelov, B. Marchand, H. Marinov, N. Moncoffre, Y. Pipon, P. Nédélec, N. Toulhoat, and D. Sillou, J. Nucl. Mater. (2012).

9 N. Djourelov, B. Marchand, H. Marinov, N. Moncoffre, Y. Pipon, N. Bérerd, P. Nédélec, L. Raimbault, and T. Epicier, J. Nucl. Mater. 443, 562 (2013).

10 R. M. Nieminen, E. Boroński, and L. J. Lantto, Phys. Rev. B 32, 1377 (1985).

11 E. Boroński and R. M. Nieminen, Phys. Rev. B 34, 3820 (1986).

12 P. E. Blöchl, Phys. Rev. B 50, 17953 (1994), URL http: //link.aps.org/doi/10.1103/PhysRevB.50.17953.

13 G. Kresse and D. Joubert, Phys. Rev. B 59, 1758 (1999), URL http://link.aps.org/doi/10.1103/PhysRevB.59. 1758.

14 N. A. W. Holzwarth, A. R. Tackett, and G. E. Matthews, Comput. Phys. Commun. 135, 329 (2001).

15 M. Torrent, F. Jollet, F. Bottin, G. Zérah, and X. Gonze, Comp. Mater. Sci. 42, 337 (2008).

16 X. Gonze, G.-M. Rignanese, M. Verstraete, J.-M. Beuke, Y. Pouillon, R. Caracas, F. Jollet, M. Torrent, G. Zérah, M. Mikami, et al., Zeit. Kristallogr. 220, 558 (2005).

17 X. Gonze, J.-M. Beuken, R. Caracas, F. Detraux, M. Fuchs, G.-M. Rignanese, L. Sindic, M. Verstraete, G. Zérah, F. Jollet, et al., Comp. Mater. Sci. 25, 478 (2002).

18 X. Gonze, B. Amadon, P.-M. Anglade, J.-M. Beuken, F. Bottin, P. Boulanger, F. Bruneval, D. Caliste, R. Caracas, M. Côté, et al., Comput. Phys. Commun. 180, 2582 (2009).

19 J. Wiktor, G. Jomard, and M. Torrent, Phys. Rev. B 92, 125113 (2015), URL http://link.aps.org/doi/10.1103/ PhysRevB.92.125113.

20 M. Alatalo, B. Barbiellini, M. Hakala, H. Kauppinen, T. Korhonen, M. J. Puska, K. Saarinen, P. Hautojärvi, and R. M. Nieminen, Phys. Rev. B 54, 2397 (1996).
21 J. Arponen and E. Pajanne, J. Phys. F: Met. Phys. 9, 2359 (1979).

22 M. J. Puska, A. P. Seitsonen, and R. M. Nieminen, Phys. Rev. B 52, 10947 (1995).

23 J. Wiktor, G. Jomard, M. Torrent, and M. Bertolus, Phys. Rev. B 87, 235207 (2013), URL http://link.aps.org/ doi/10.1103/PhysRevB.87.235207.

24 J. Wiktor, G. Jomard, and M. Bertolus, Nucl. Instrum. Meth. B 327, 63 (2014), URL http: //www.sciencedirect.com/science/article/pii/ S0168583X14001529.

25 J. Wiktor, X. Kerbiriou, G. Jomard, S. Esnouf, M.-F. Barthe, and M. Bertolus, Phys. Rev. B 89, 155203 (2014).

26 J. P. Perdew, K. Burke, and M. Ernzerhof, Phys. Rev. Lett. 77, 3865 (1996), URL http://link.aps.org/doi/ 10.1103/PhysRevLett.77.3865.

27 A. I. Liechtenstein, V. I. Anisimov, and J. Zaanen, Phys. Rev. B 52, R5467 (1995), URL http://link.aps.org/ doi/10.1103/PhysRevB.52.R5467.

28 S. L. Dudarev, D. N. Manh, and A. P. Sutton, Phil. Mag. B 75, 613 (1997).

29 B. Dorado, G. Jomard, M. Freyss, and M. Bertolus, Phys. Rev. B 82, 035114 (2010).

30 B. Dorado, D. A. Andersson, C. R. Stanek, M. Bertolus, B. P. Uberuaga, G. Martin, M. Freyss, and P. Garcia, Phys. Rev. B 86, 035110 (2012), URL http://link.aps.org/ doi/10.1103/PhysRevB.86.035110.

31 B. Dorado, B. Amadon, M. Freyss, and M. Bertolus, Phys. Rev. B 79, 235125 (2009), URL http://link.aps.org/ doi/10.1103/PhysRevB.79.235125.

32 E. Vathonne, J. Wiktor, M. Freyss, G. Jomard, and M. Bertolus, J. Phys.: Condens. Mat. 26, 325501 (2014).

33 A. Kotani and T. Yamazaki, Prog. Theor. Phys. Suppl. 108, 117 (1992).

34 G. Jomard, B. Amadon, F. m. c. Bottin, and M. Torrent, Phys. Rev. B 78, 075125 (2008), URL http://link.aps. org/doi/10.1103/PhysRevB.78.075125.

35 B. Amadon, F. Jollet, and M. Torrent, Phys. Rev. B 77, 155104 (2008), URL http://link.aps.org/doi/10.1103/ PhysRevB.77.155104.

36 R. Laskowski, G. K. H. Madsen, P. Blaha, and K. Schwarz, Phys. Rev. B 69, 140408 (2004), URL http://link.aps. org/doi/10.1103/PhysRevB.69.140408.

37 P. Santini, R. Lemanski, and P. Erdős, Advances in Physics 48, 537 (1999).

38 P. Santini, S. Carretta, G. Amoretti, R. Caciuffo, N. Magnani, and G. H. Lander, Reviews of Modern Physics 81, 807 (2009).

39 B. Dorado and P. Garcia, Physical Review B 87, 195139 (2013).

40 T. Korhonen, M. J. Puska, and R. M. Nieminen, Phys. Rev. B 54, 15016 (1996).

41 A. Kokalj, J. Mol. Graph. Model. 17, 176 (1999).

42 A. Kokalj, Comput. Mat. Sci. 28, 155 (2003). 